\title{
PROBABILISTIC INVESTIGATIONS ON THE EXPLOSION OF SOLUTIONS OF THE KAC EQUATION WITH INFINITE ENERGY INITIAL DISTRIBUTION
}

\author{
ERIC CARLEN, ${ }^{*}$ Rutgers University \\ ESTER GABETTA, ${ }^{* *}$ Università degli Studi di Pavia \\ EUGENIO REGAZZINI, ${ }^{* *} * * *$ Università degli Studi di Pavia and IMATI-CNR
}

\begin{abstract}
Gabetta and Regazzini (2006b) have shown that finiteness of the initial energy (second moment) is necessary and sufficient for the solution of the Kac's model Boltzmann equation to converge weakly ( $C_{b}$-convergence) to a probability measure on $\mathbb{R}$. Here, we complement this result by providing a detailed analysis of what does actually happen when the initial energy is infinite. In particular, we prove that such a solution converges vaguely $\left(C_{0}\right.$-convergence) to the zero measure (which is identically 0 on the Borel sets of $\mathbb{R}$ ). More precisely, we prove that the total mass of the limiting distribution splits into two equal masses (of value $\frac{1}{2}$ each), and we provide quantitative estimates on the rate at which such a phenomenon takes place. The methods employed in the proofs also apply in the context of sums of weighted independent and identically distributed random variables $\tilde{x}_{1}, \tilde{x}_{2}, \ldots$, where these random variables have an infinite second moment and zero mean. Then, with $T_{n}:=\sum_{j=1}^{v_{n}} \lambda_{j, n} \tilde{x}_{j}$, with $\max _{1 \leq j \leq v_{n}} \lambda_{j, n} \rightarrow 0($ as $n \rightarrow+\infty)$, and $\sum_{j=1}^{v_{n}} \lambda_{j, n}^{2}=1, n=1,2, \ldots$, the classical central limit theorem suggests that $T$ should in some sense converge to a 'normal random variable of infinite variance'. Again, in this setting we prove quantitative estimates on the rate at which the mass splits into adherent masses to $-\infty$ and $+\infty$, or to $\infty$, that are analogous to those we have obtained for the Kac equation. Although the setting in this case is quite classical, we have not uncovered any previous results of a similar type.
\end{abstract}

Keywords: Boltzmann equation; central limit theorem

2000 Mathematics Subject Classification: Primary 60F05

Secondary $82 \mathrm{C} 40$

\section{Presentation}

The Kac model Boltzmann equation, under the usual hypothesis that the initial velocity probability distribution $\mu_{0}$ is absolutely continuous with density function $f_{0}$, can be written as

$$
\begin{gathered}
\frac{\partial}{\partial t} f(v, t)=\frac{1}{2 \pi} \int_{\mathbb{R} \times[0,2 \pi)}(f(v \cos \theta-w \sin \theta, t) f(v \sin \theta+w \cos \theta, t) \\
-f(v, t) f(w, t)) \mathrm{d} w \mathrm{~d} \theta,
\end{gathered}
$$

\footnotetext{
Received 31 May 2007; revision received 7 January 2008.

* Postal address: Department of Mathematics, Rutgers University, 110 Frelinghuysen Road, Piscataway, NJ 088548019, USA.

** Postal address: Dipartimento di Matematica "F. Casorati”, Università degli Studi di Pavia, via Ferrata 1, I-27100 Pavia, Italy.

*** Email address: eugenio.regazzini@unipv.it
} 


$$
f(v, 0)=f_{0}(v), \quad t>0, v \in \mathbb{R},
$$

where $f(\cdot, t)$ denotes the probability density function of the velocity at time $t$. See Kac (1956), (1959). In terms of the Fourier transforms $\varphi_{0}$ and $\varphi(\cdot, t)$ of $f_{0}$ and $f(\cdot, t)$, respectively, the previous equation becomes

$$
\begin{gathered}
\frac{\partial}{\partial t} \varphi(\xi, t)=\frac{1}{2 \pi} \int_{0}^{2 \pi} \varphi(\xi \sin \theta, t) \varphi(\xi \cos \theta, t) \mathrm{d} \theta-\varphi(\xi, t), \\
\varphi(\xi, 0)=\varphi_{0}(\xi), \quad t>0, \xi \in \mathbb{R} .
\end{gathered}
$$

See Bobylev (1984). In the present paper we mainly deal with (1), since it is valid even when $\varphi_{0}$ is the Fourier-Stieltjes transform (characteristic function) of any, not necessarily absolutely continuous, initial distribution $\mu_{0}$.

Equation (1) has a unique solution that can be expressed by Wild sums. See Wild (1951). More precisely,

$$
\varphi(\xi, t)=\sum_{n \geq 1} \mathrm{e}^{-t}\left(1-\mathrm{e}^{-t}\right)^{n-1} \hat{Q}_{n}^{+}\left(\xi ; \varphi_{0}\right), \quad t \geq 0, \xi \in \mathbb{R},
$$

with $\hat{Q}_{n}^{+}$determined recursively according to

$$
\hat{Q}_{n}^{+}\left(\xi ; \varphi_{0}\right)=\frac{1}{n-1} \sum_{j=1}^{n-1} \hat{Q}_{n-j}^{+}\left(\cdot ; \varphi_{0}\right) \circ \hat{Q}_{j}^{+}\left(\cdot ; \varphi_{0}\right)(\xi), \quad n=2,3, \ldots,
$$

with $\hat{Q}_{1}^{+}:=\varphi_{0}$ and 'o' defined as follows: given any pair of characteristic functions $\hat{g}_{1}$ and $\hat{g}_{2}$, $\hat{g}_{1} \circ \hat{g}_{2}$ is defined by

$$
\hat{g}_{1} \circ \hat{g}_{2}(\xi)=\frac{1}{2 \pi} \int_{0}^{2 \pi} \hat{g}_{1}(\xi \cos \theta) \hat{g}_{2}(\xi \sin \theta) \mathrm{d} \theta, \quad \xi \in \mathbb{R} .
$$

Gabetta and Regazzini (2006b) proved that finiteness of the initial energy (second moment) is necessary and sufficient for $\mu(\cdot, t)$ — the probability measure corresponding to $\varphi(\cdot, t)$ - to converge weakly ( $C_{b}$-convergence) to a probability measure on $\mathbb{R}$ as $t$ goes to $+\infty$. According to standard notation, throughout the paper, $C(\mathbb{R})$ and $C_{b}(\mathbb{R})$ will respectively stand for the class of continuous real-valued functions and the class of bounded and continuous real-valued functions on $\mathbb{R}$. While the sufficiency of the above condition is well known, there does not seem to have been any proof of its necessity, even though it is intuitively clear on physical grounds.

Taking this statement, of a qualitative nature, as a starting point, in the present study we set out to analyze the vague convergence of $\mu(\cdot, t)$ to the null measure when

$$
\int_{\mathbb{R}} v^{2} \mu_{0}(\mathrm{~d} v)=+\infty
$$

Before describing the results we obtain in this study, let us briefly comment on its motivations. From a mathematical perspective, the Wild sum provides a canonical solution of the Kac model Boltzmann equation regardless of whether or not the second moment is finite. While the precise long-time behavior of solutions with finite energy initial data, i.e. finite second moment initial data, is well known, very little has been proved in the infinite energy case. It is of clear mathematical interest to redress this situation. 
From a physical perspective, the case of infinite energy initial data is significant for the following reason. The equation of ultimate interest is the spatially inhomogeneous Boltzmann equation, which in the Kac model case describes the evolution of a probability density $f(x, v, t)$ on the phase space $\mathbb{R}^{2}$. The time-dependent probability density $f(x, v, t)$ characterizes the joint distribution, at time $t$, of the position and of the velocity of a molecule. In this spatially dependent setting there are two evolution mechanisms at work. One is the collision process, which happens locally at each $x$, and is described by the spatially homogeneous equation studied here. The other is streaming, where molecules with velocity $v$ move according to their velocity between collisions. Combining these effects, we have the spatially inhomogeneous Kac equation

$$
\begin{aligned}
\frac{\partial}{\partial t} f(x, v, t)+v \frac{\partial}{\partial x} f(x, v, t)=\frac{1}{2 \pi} \int_{\mathbb{R} \times[0,2 \pi)} & (f(x, v \cos \theta-w \sin \theta, t) \\
& \times f(x, v \sin \theta+w \cos \theta, t) \\
& -f(x, v, t) f(x, w, t)) \mathrm{d} w \mathrm{~d} \theta, \\
f(x, v, 0)=f_{0}(x, v), \quad t & >0, v \in \mathbb{R} .
\end{aligned}
$$

The physically interesting data for this equation does satisfy the finite energy condition,

$$
\int_{\mathbb{R}^{2}} v^{2} f_{0}(x, v) \mathrm{d} x \mathrm{~d} v<\infty .
$$

Still, this does not imply the finiteness of the conditional variance $\theta_{0}(x)$ of the velocity given the position $x$, evaluated according to $f_{0}$. The physical interpretation of $\theta_{0}(x)$ is the initial temperature at $x$. Likewise, the conditional variance $\theta(x, t)$ of the velocity given the position $x$ at time $t$ represents the local temperature at $x$ and $t$. Even if the total energy is finite, the temperature $\theta(x, t)$ may well have singularities, so that $\theta(x, t)$ may be infinite for some $x$ and $t$. In fact, 'hot spots' may develop in time even if they are not present initially.

The spatially homogeneous equation studied here describes the evolution of the conditional velocity distribution under the influence of collisions alone, for example in a 'splitting method', where one solves the spatially inhomogeneous equation by alternately running the streaming process and collision process in alternate small time steps. The theorems we prove below show that the hot spots will quickly disperse. As we shall show, because of the collisions at $x$, all of the molecules at the hot spot quickly pick up very high velocities, isotropically distributed, which then 'explode away' from the hot spot, owing to the streaming and their high velocities. Note that our use of the term 'explosion' here is literal and physical: it is not to be confused with the common use in the theory of stochastic processes, concerning the loss of probability at finite times. That does not happen here. What does happen is that all of the molecules involved in the hot spot rush away from it at high speeds and isotropically, which is what happens in an actual explosion.

Hopefully, this brief discussion of the spatially inhomogeneous equation explains the physical motivation for our study, and we now return to the study of the spatially homogeneous equation for infinite energy initial data.

Recall that a sequence $\left(\mu_{n}\right)_{n \geq 1}$ of probability measures on $(\mathbb{R}, \mathcal{B}(\mathbb{R}))$ is said to be convergent vaguely to a measure $\mu$ if $\int_{\mathbb{R}} f(x) \mu_{n}(\mathrm{~d} x) \rightarrow \int_{\mathbb{R}} f(x) \mu(\mathrm{d} x)$ for any $f$ in $C_{0}(\mathbb{R}):=\{f \in$ $C(\mathbb{R}): f$ vanishes at $\infty\}$. With respect to our specific case, vague convergence of $\mu(\cdot, t)$ to the null measure is tantamount to asserting that

$$
\mu(I, t) \rightarrow 0, \quad t \rightarrow+\infty, I \text { is any bounded interval. }
$$


In point of fact, Theorem 1, below, states that if the initial energy is infinite then the total mass of the limiting distribution (in the weak sense of de Finetti) splits into two equal masses (of value $\frac{1}{2}$ each) which adhere to $-\infty$ and $+\infty$, respectively. See de Finetti (1970, Section 6.4).

Above all, we aim at providing a quantitative estimation of the corresponding rate of convergence. To this end, it is worth introducing truncated moments

$$
m_{k}(L):=\int_{[-L, L]} v^{k} \mu_{0}(\mathrm{~d} v), \quad k=0,1, \ldots, L \in \mathbb{R},
$$

of the initial probability measure $\mu_{0}$ in problem (1) or (2).

Theorem 1. Set $\tau_{1}:=(-\infty,-R], \tau_{2}:=[R, \infty)$, and set

$$
L_{t}:=\exp \left(t\left(1-\frac{8}{3 \pi}\right)\right) \text {. }
$$

Assume that $\mu_{0}$ satisfies (4), and let $\eta$ be a fixed number in $(0,1)$. Then, there is a time $t_{\eta}$ such that, for every $t \geq t_{\eta}, \eta \vee\left(1-\eta^{2}\right)<m_{0}\left(L_{t}\right)<1$ is valid and, for $1 \leq i \neq j \leq 2$,

$$
\frac{1}{2}-A(t)+B_{i, j}(t) \leq \mu\left(\tau_{i}, t\right) \leq \frac{1}{2}+B_{i, j}(t)
$$

holds for every $R \geq 2\left(\left(m_{0}\left(L_{t}\right)-\eta\right)(2-\sqrt{2})\right)^{-1}$ with

$$
A(t):=\frac{R}{m_{2}\left(L_{t}\right)^{1 / 2}\left(m_{0}\left(L_{t}\right)-\eta\right)^{1 / 2}}+\frac{1}{2} \mathrm{e}^{-t / 4}
$$

and

$$
B_{i, j}(t):=\frac{1}{2} \mathrm{e}^{-t}\left(\mu_{0}\left(\tau_{i}\right)-\mu_{0}\left(\tau_{j}\right)\right) .
$$

The methods used in Section 3, to prove Theorem 1, can also be applied to the study of the asymptotic behavior of the probability distribution of the sum

$$
T_{n}:=\sum_{j=1}^{v_{n}} \lambda_{j, n} \tilde{x}_{j}
$$

when $\tilde{x}_{1}, \tilde{x}_{2}, \ldots$ are independent and identically distributed random variables and, for each $n$, $\left(\lambda_{j, n}\right)_{j=1, \ldots, v_{n}}$ are nonnegative numbers and $v_{n}$ are positive integers such that

$$
\lambda_{(n)}:=\max _{1 \leq j \leq \nu_{n}} \lambda_{j, n} \rightarrow 0 \quad \text { as } n \rightarrow+\infty
$$

and

$$
\sum_{j=1}^{v_{n}} \lambda_{j, n}^{2}=1, \quad n=1,2, \ldots
$$

Solutions of the above problem can be easily found by resorting to the central limit theorem if the variance of each $\tilde{x}_{j}$ is finite. Instead, when the variance is infinite, there seems to have been only partial and isolated answers like, for example, an example of Crimaldi (2002) in which $\lambda_{j, n}=1 / \sqrt{n}, v_{n}=n$, and the common distribution of the $\tilde{x}_{j}$ meets a few extra conditions. Assume that the $\tilde{x}_{j}$ s are defined on some space equipped with probability P. We start with the case in which the common distribution of the $\tilde{x}_{j}$ s is symmetric. 
Corollary 1. Let the random variables $\tilde{x}_{j}$, in (5), have symmetric common probability distribution $\mu_{0}$ satisfying (4). Moreover, let $\left(\lambda_{j, n}\right)_{n \geq 1}$ satisfy (6) and (7). Then, for every $\eta$ in $(0,1)$, there is $N_{\eta}$ such that $\eta \vee\left(1-\eta^{2}\right)<m_{0}\left(L_{n}\right)<1$ holds for $L_{n}:=\lambda_{(n)}^{-1}$ with $n \geq N_{\eta}$. Furthermore, under these very same conditions, we have

$$
\frac{1}{2}-A(n) \leq \mathrm{P}\left\{T_{n} \leq-R\right\}=\mathrm{P}\left\{T_{n} \geq R\right\} \leq \frac{1}{2}
$$

with

$$
A(n):=\frac{R}{\sqrt{m_{2}\left(L_{n}\right)\left(m_{0}\left(L_{n}\right)-\eta\right)}}+\frac{1}{2} \lambda_{(n)}^{2},
$$

provided that $R \geq 2\left(\left(m_{0}\left(L_{n}\right)-\eta\right)(2-\sqrt{2})\right)^{-1}$.

From a slight modification of the proof of the previous statements we obtain an extension of the thesis of the corollary to arbitrary $\mu_{0}$ satisfying (4). In fact, if $\mu_{0}$ is not symmetric, we succeed in estimating the velocity in which the mass is shifted to $\{-\infty,+\infty\}$ : without further conditions, we are not able to distinguish the behavior at $-\infty$ from that at $+\infty$.

Proposition 1. Let the random variables $\tilde{x}_{j}$, in (5), have common probability distribution $\mu_{0}$ satisfying (4). Moreover, let $\left(\lambda_{j, n}\right)_{n \geq 1}$ satisfy (6) and (7). Then, for every $\eta$ in $(0,1)$, there is $N_{\eta}$ such that $\eta \vee\left(1-\eta^{2}\right)<m_{0}\left(L_{n}\right)<1$ and

$$
\frac{m_{2}\left(L_{n}\right)}{m_{0}\left(L_{n}\right)}-\left(\frac{m_{1}\left(L_{n}\right)}{m_{0}\left(L_{n}\right)}\right)^{2} \geq(1-\eta) m_{2}\left(L_{n}\right)
$$

hold for every $L_{n}:=\lambda_{(n)}^{-1}$ with $n \geq N_{\eta}$. Furthermore, under these very same conditions, we have

$$
\mathrm{P}\left\{\left|T_{n}\right| \leq R\right\} \leq \frac{2 R}{m_{2}\left(L_{n}\right)^{1 / 2}\left(m_{0}-\eta\right)}+\lambda_{(n)}^{2},
$$

provided that $R \geq 4\left(\left(m_{0}\left(L_{n}\right)-\eta\right)(2-\sqrt{2})\right)^{-1}$.

The present authors have also studied the case of the spatially homogeneous Boltzmann equation for Maxwellian molecules when the initial energy is infinite. See Carlen et al. (2007). The analysis is, however, quite different, so there is little methodological overlap with the present paper, and the bounds obtained in the present setting are somewhat simpler and more explicit.

With this, the presentation of the main results ends. Their proofs are presented in Section 3. However, in Section 2 we first explain the general ideas of the proofs, and we recall a few well-known statements to be used in Section 3.

\section{Preliminaries to proofs}

There have been several previous investigations of the Kac equation using probabilistic methods. For example, Tanaka (1973), Murata and Tanaka (1974), and Tanaka (1978) proved a contraction property in the 2-Wasserstein metric. However, this is only defined on probability measures with finite second moments. For other probabilistic investigations, see McKean (1963) and Carlen et al. (2000), (2005). Here, the infinite energy (variance) setting requires a new approach.

The argument used to prove Theorem 1 rests on two main ideas. The former stems from the remark that the Wild series, as rewritten in McKean (1966) and McKean (1967), highlights that 
$\mu(\cdot, t)$ can be viewed as a probability distribution of a linear combination of a random number (with probability law depending on $t$ ) of identically distributed random variables with common distribution $\mu_{0}$ and random coefficients. The latter relates to the fact that $\mu_{0}$ can be thought of as a probability law of

$$
\alpha Y+(1-\alpha) Z
$$

when $\alpha, Y$, and $Z$ are stochastically independent random variables defined in such a way that $\alpha$ belongs to $\{0,1\}$ and takes value 1 with probability $m_{0}(L)$ in $(0,1)$ for a suitable choice of $L$, and $Y$ and $Z$ have distribution (on the Borel class of $\mathbb{R}$ )

$$
\underline{\mu}_{L}(\cdot):=\frac{1}{m_{0}(L)} \mu_{0}([-L, L] \cap \cdot)
$$

and

$$
\bar{\mu}_{L}(\cdot):=\frac{1}{1-m_{0}(L)} \mu_{0}\left([-L, L]^{c} \cap \cdot\right)
$$

respectively.

For the sake of explanation, we start by recalling the theorem of McKean which states that $\hat{Q}_{n}^{+}$(see (3)) can be written as

$$
\hat{Q}_{n}^{+}\left(\xi ; \varphi_{0}\right)=\sum_{\gamma \in G(n)} p_{n}(\gamma) C_{\gamma}\left(\xi ; \varphi_{0}\right), \quad \xi \in \mathbb{R}, n=2,3, \ldots,
$$

where

- $G(n)$ is the set of all trees $\gamma$ with $n$ leaves in which each node has either 0 or 2 'children'; the leaves of these trees are labeled, from left to right, with the first $n$ natural numbers and the nodes are labeled in such a way that 1 designates the root node at the top, 2 is attached to the minimal node at level 1 , and so on;

- $p_{n}(\cdot)$ is a specific probability on $G(n)$;

- $C_{\gamma}\left(\cdot ; \varphi_{0}\right)$, called the $n$-fold product of $\varphi_{0}$ with itself, performed according to $\gamma$, is given by

$$
C_{\gamma}\left(\xi ; \varphi_{0}\right)=\int_{[0,2 \pi)^{\mathbb{N}}}\left(\prod_{l \in \gamma} \varphi_{0}\left(\pi_{l} \xi\right)\right) u^{\otimes \mathbb{N}}(\mathrm{d} \theta), \quad \gamma \in G:=\bigcup_{n \geq 1} G(n), \xi \in \mathbb{R}
$$

with $\pi_{l} \equiv 1$ if $\gamma$ is the sole element of $G(1)$, where $u^{\otimes \mathbb{N}}$ is the probability measure on the Borel subsets of $[0,2 \pi)^{\mathbb{N}}$ which makes the coordinates stochastically independent with the same uniform continuous distribution on $[0,2 \pi)$.

Angle $\theta_{1}$ is associated with node 1 of $\gamma, \theta_{2}$ is associated with node 2 of $\gamma$, and so on. Now, for any leaf $l$ of $\gamma$ in $G(n)$, look at the path which connects $l$ and the 'root' node. It consists of $\delta_{l}$ steps ( $\delta_{l}$ is said to be the depth of $l$ ): the first one from $j$ to its 'parent' node, the second one from the 'parent' to the 'grandparent' of $l$, etc. After singling out the angles, associated with the nodes met by the path, say $\theta_{\sigma(1)}, \ldots, \theta_{\sigma\left(\delta_{l}\right)}, \sigma(1) \equiv 1$, we define $\pi_{l}$ to be the product of $\alpha_{1}(\sigma(1)) \cdots \alpha_{\delta_{l}}\left(\sigma\left(\delta_{l}\right)\right)$, where $\alpha_{\delta_{l}}$ equals $\cos \theta_{\sigma\left(\delta_{l}\right)}$ if $l$ is a 'left child' or $\sin \theta_{\sigma\left(\delta_{l}\right)}$ if $l$ is a 'right child'; $\alpha_{\delta_{l}-1}$ equals $\cos \theta_{\sigma\left(\delta_{l}-1\right)}$ or $\sin \theta_{\sigma\left(\delta_{l}-1\right)}$ depending on whether the parent of $l$ is, in its turn, a 'left child' or a 'right child', and so on. Now, for each tree $\gamma$ in $G$, set $s(\gamma)=\sum_{l \in \gamma} \pi_{l}^{2}$, with the proviso that $s(\gamma)=1$ if $\gamma$ is the sole tree in $G(1)$. Then

$$
s(\gamma)=1, \quad \gamma \in G,
$$


holds true. This fact can be proved by induction. It is trivially true for $\gamma$ in $G(1)$. Next, assume that it is valid for every tree in $\bigcup_{k=1}^{n} G(k)$. Given any tree in $G(n+1)$, remove its root node to obtain two subtrees $\gamma_{1}$ and $\gamma_{\mathrm{r}}$, the 'left' and the 'right' subtrees, respectively, which belong to $\bigcup_{k=1}^{n} G(k)$. Moreover, we can write $s(\gamma)=\cos ^{2} \theta_{1} s\left(\gamma_{1}\right)+\sin ^{2} \theta_{1} s\left(\gamma_{\mathrm{r}}\right)$ which, together with the inductive hypothesis (according to which $s\left(\gamma_{1}\right)=s\left(\gamma_{\mathrm{r}}\right)=1$ ), yields (10).

Next, we recall the argument developed in Gabetta and Regazzini (2006b) to show that (2) represents the characteristic function of a sum of random variables defined on the probability space we are about to define. Let

$$
\Omega=(\mathbb{N} \times G) \times[0,2 \pi)^{\mathbb{N}} \times\{0,1\}^{\mathbb{N}} \times\left(\mathbb{R}^{2}\right)^{\mathbb{N}},
$$

denote the coordinate maps of $\Omega$ by

$$
\left(\tilde{v}_{t}, \tilde{\gamma}\right), \quad\left(\tilde{\theta}_{1}, \tilde{\theta}_{2}, \ldots\right), \quad\left(\tilde{\alpha}_{1}, \tilde{\alpha}_{2}, \ldots\right), \quad\left(\left(\tilde{Y}_{1}, \tilde{Z}_{1}\right),\left(\tilde{Y}_{2}, \tilde{Z}_{2}\right), \ldots\right),
$$

and consider the $\sigma$-algebra

$$
\mathcal{F}=\mathcal{P}(\mathbb{N} \times G) \otimes \mathscr{B}\left([0,2 \pi)^{\mathbb{N}}\right) \otimes \mathscr{B}\left(\{0,1\}^{\mathbb{N}}\right) \otimes \mathscr{B}\left(\left(\mathbb{R}^{2}\right)^{\mathbb{N}}\right),
$$

where, for any set $S, \mathcal{P}(S)$ stands for the power set of $S$. At this stage equip $(\Omega, \mathcal{F})$ with the probability measure $\mathrm{P}_{t}$ characterized by assigning the value

$$
\mathrm{P}_{t}(M \times T \times A \times D \times E)=\sum_{n \in M} \sum_{\gamma \in T} q_{t}(n) \mathbb{I}_{G(n)} p_{n}(\gamma) u^{\otimes \mathbb{N}}(A) \beta(D)\left(\underline{\mu}_{L} \times \bar{\mu}_{L}\right)^{\otimes \mathbb{N}}(E)
$$

to any rectangle with $M \subset \mathbb{N}, T \subset G, A \in \mathcal{B}\left([0,2 \pi)^{\mathbb{N}}\right), D \in \mathcal{B}\left(\{0,1\}^{\mathbb{N}}\right)$, and $E \in$ $\mathscr{B}\left(\left(\mathbb{R}^{2}\right)^{\mathbb{N}}\right)$, where $\beta$ is the law of a Bernoulli sequence with probability of success $m_{0}(L)$, $q_{t}(n)=\mathrm{e}^{-t}\left(1-\mathrm{e}^{-t}\right)^{n-1}$ for $n=1,2, \ldots$, and $\mathbb{I}_{A}$ denotes the indicator of $A$.

In view of the independence induced by $\mathrm{P}_{t}$ among the coordinate maps, we can say that the random variables

$$
\tilde{\alpha}_{i} \tilde{Y}_{i}+\left(1-\tilde{\alpha}_{i}\right) \tilde{Z}_{i}, \quad i=1,2, \ldots,
$$

are independent with the same distribution $\mu_{0}$. Indeed, for the characteristic function $\phi_{i}$ of $\tilde{\alpha}_{i} \tilde{Y}_{i}+\left(1-\tilde{\alpha}_{i}\right) \tilde{Z}_{i}, i=1,2, \ldots$, we obtain

$$
\phi_{i}(\xi)=\left(1-m_{0}(L)\right) \phi_{\tilde{Z}_{i}}(\xi)+m_{0}(L) \phi_{\tilde{Y}_{i}}(\xi)
$$

where

$$
\phi_{\tilde{Z}_{i}}(\xi)=\int_{[-L, L]^{c}} \frac{\mathrm{e}^{i \xi x} \mu_{0}(\mathrm{~d} x)}{1-\mu_{0}(L)}, \quad \phi_{\tilde{Y}_{i}}(\xi)=\int_{[-L, L]} \frac{\mathrm{e}^{i \xi x} \mu_{0}(\mathrm{~d} x)}{m_{0}(L)}, \quad \text { for every } i,
$$

which yields $\phi_{i}=\phi_{0}, i=1,2, \ldots$

At this stage, observe that the characteristic function $\phi_{\tilde{V}_{t}}$ of

$$
\tilde{V}_{t}:=\sum_{l=1}^{\tilde{v}_{t}} \pi_{l}\left(\tilde{\alpha}_{l} \tilde{Y}_{l}+\left(1-\tilde{\alpha}_{l}\right) \tilde{Z}_{l}\right)
$$

can be written, in view of the definition of $\mathrm{P}_{t}$, as

$$
\phi_{\tilde{V}_{t}}(\xi)=\sum_{n \geq 1} \mathrm{e}^{-t}\left(1-\mathrm{e}^{-t}\right)^{n-1} \sum_{\gamma \in G(n)} p_{n}(\gamma) \int_{[0,2 \pi)^{\mathbb{N}}}\left(\prod_{l \in \gamma} \phi_{l}\left(\pi_{l} \xi\right)\right) u^{\otimes \mathbb{N}}(\mathrm{d} \theta),
$$


which, combining the fact that $\phi_{l}=\phi_{0}$ for every $l$ with (8)-(9), turns out to be the same as $\phi(\cdot, t)$ in (2), which is tantamount to saying that the distribution of $\tilde{V}_{t}$ is $\mu(\cdot, t)$.

The following identities from Gabetta and Regazzini (2006a) are frequently used in the rest of this paper:

$$
\begin{aligned}
\mathrm{E}_{t}\left(\sum_{l=1}^{\tilde{v}_{t}}\left(\frac{c}{2}\right)^{\delta_{l}(\tilde{\gamma})} \mid \tilde{v}_{t}\right) & =\frac{\Gamma\left(c+\tilde{v}_{t}-1\right)}{\Gamma(c) \Gamma\left(\tilde{v}_{t}\right)}, \\
\mathrm{E}_{t}\left(\sum_{l=1}^{\tilde{v}_{t}}\left(\frac{c}{2}\right)^{\delta_{l}(\tilde{\gamma})}\right) & =\mathrm{e}^{-t(1-c)},
\end{aligned}
$$

where $c$ is any strictly positive number and $\delta_{l}(\tilde{\gamma})$ is the depth of leaf $l$ in tree $\tilde{\gamma}$. Moreover,

$$
\begin{gathered}
\mathrm{E}_{t}\left(\sum_{l=1}^{\tilde{v}_{t}}\left|\pi_{l}\right|^{m} \mid \tilde{v}_{t}\right)=\frac{\Gamma\left(2 \beta_{m}+\tilde{v}_{t}-1\right)}{\Gamma\left(2 \beta_{m}\right) \Gamma\left(\tilde{v}_{t}\right)}, \\
\mathrm{E}_{t}\left(\sum_{l=1}^{\tilde{v}_{t}}\left|\pi_{l}\right|^{m}\right)=\mathrm{e}^{-t\left(1-2 \beta_{m}\right)},
\end{gathered}
$$

with

$$
\beta_{m}=\frac{1}{2 \pi} \int_{0}^{2 \pi}|\sin \theta|^{m} \mathrm{~d} \theta, \quad m=0,1,2, \ldots
$$

Finally, we recall that (4) implies that, for every $\eta$ in $(0,1)$, there is $L_{\eta}$ such that

$$
\frac{m_{2}(L)}{m_{0}(L)}-\left(\frac{m_{1}(L)}{m_{0}(L)}\right)^{2} \geq(1-\eta) m_{2}(L)
$$

holds true for every $L>L_{\eta}$. A proof can be found in Khintchine (1935) on the characterization of the domain of attraction of a Gaussian law. See also Theorem 1 in Section 35 of Gnedenko and Kolmogorov (1954).

\section{Proofs}

Set

$$
\begin{gathered}
\tilde{S}_{1, t}:=\sum_{l=1}^{\tilde{v}_{t}} \pi_{l} \tilde{\alpha}_{l} \tilde{Y}_{l}, \quad \tilde{S}_{2, t}:=\sum_{l=1}^{\tilde{v}_{t}} \pi_{l}\left(1-\tilde{\alpha}_{l}\right) \tilde{Z}_{l}, \\
\tilde{s}_{t}:=\left(\tilde{v}_{t}, \tilde{\gamma}, \tilde{\theta}, \tilde{\alpha}\right), \quad \tilde{\theta}:=\left(\tilde{\theta}_{1}, \tilde{\theta}_{2}, \ldots\right), \quad \tilde{\alpha}:=\left(\tilde{\alpha}_{1}, \tilde{\alpha}_{2}, \ldots\right) .
\end{gathered}
$$

For the sake of computational simplicity, it is worth recalling that $\hat{Q}_{n}^{+}$coincides for any $n=$ $2,3, \ldots$ with the Fourier-Stieltjes transform of the $n$-fold Wild convolution when $\phi_{0}$ is replaced by its real part. See McKean (1966, p. 359). Then, the probability distribution of $\tilde{V}_{t}$ can be written as a sum of (i) the distribution of $\tilde{V}_{t}$, when the initial measure $\mu_{0}$ is replaced by its even part (the probability $\bar{\mu}_{0}$ whose Fourier-Stieltjes transform is the real part of $\phi_{0}$ ); and (ii) $\mathrm{e}^{-t}\left(\mu-\bar{\mu}_{0}\right)$. Throughout this section, $\overline{\mathrm{P}}_{t}$ will denote the probability distribution defined in the same way as $\mathrm{P}_{t}$ with $\mu_{0}$ replaced by $\bar{\mu}_{0}$. Thus,

$$
\begin{aligned}
\mu_{t}(B, t) & =\mathrm{P}_{t}\left(\tilde{V}_{t} \in B\right) \quad(B \in \mathcal{B}(\mathbb{R})) \\
& =\overline{\mathrm{P}}_{t}\left(\tilde{V}_{t} \in B\right)+\mathrm{e}^{-t}\left(\mu_{0}(B)-\bar{\mu}_{0}(B)\right) .
\end{aligned}
$$


Observe that both $\underline{\mu}_{L}$ and $\bar{\mu}_{L}$ turn out to be symmetric when $\bar{\mu}_{0}$ takes the place of $\mu_{0}$. Then,

$$
\overline{\mathrm{E}}_{t}\left(\tilde{S}_{1, t} \mid \tilde{s}_{t}\right)=0 \quad \text { and } \quad \overline{\operatorname{var}}_{t}\left(\tilde{S}_{1, t} \mid \tilde{s}_{t}\right)=b\left(\tilde{s}_{t}\right)^{2} M_{2}(L)
$$

where

$$
b\left(\tilde{s}_{t}\right)^{2}:=\sum_{l=1}^{\tilde{v}_{t}} \pi_{l}^{2} \tilde{\alpha}_{l}^{2}, \quad M_{k}(L):=\int_{\mathbb{R}}|x|^{k} \underline{\mu}_{l}(\mathrm{~d} x)=\frac{m_{k}(L)}{m_{0}(L)}, \quad k=1,2, \ldots
$$

Moreover, let $F_{1, t}$ and $F_{2, t}$ denote the conditional probability distribution functions of $\tilde{S}_{1, t}$ and $\tilde{S}_{2, t}$, respectively, given $\tilde{s}_{t}$, and let $F_{1, t}^{*}$ denote the conditional probability distribution function of $\tilde{S}_{1, t}^{*}$ given $\tilde{s}_{t}$, where, for any $y$ in $\mathbb{R}$,

$$
y^{*}:=\frac{y}{\bar{M}_{2}(L)^{1 / 2} b\left(\tilde{s}_{t}\right)} .
$$

To prove Theorem 1, use conditional independence and (11) to write, for every positive $R$,

$$
\begin{aligned}
\overline{\mathrm{P}}_{t}\left(\left|\tilde{V}_{t}\right|>R\right) & =\overline{\mathrm{E}}_{t}\left(\overline{\mathrm{P}}_{t}\left(\tilde{S}_{1, t}+\tilde{S}_{2, t}<-R \mid \tilde{s}_{t}\right)\right)+\overline{\mathrm{E}}_{t}\left(\overline{\mathrm{P}}_{t}\left(\tilde{S}_{1, t}+\tilde{S}_{2, t}>R \mid \tilde{s}_{t}\right)\right) \\
& =2 \overline{\mathrm{P}}_{t}\left(\tilde{S}_{1, t}+\tilde{S}_{2, t}<-R\right) \quad(\text { from symmetry }) .
\end{aligned}
$$

Conversely,

$$
\overline{\mathrm{P}}_{t}\left(\left|\tilde{V}_{t}\right|>R\right)=1-\overline{\mathrm{P}}_{t}\left(\left|\tilde{V}_{t}\right| \leq R\right)
$$

and

$$
\begin{aligned}
\overline{\mathrm{P}}_{t}\left(\left|\tilde{V}_{t}\right| \leq R\right) & =\overline{\mathrm{E}}_{t}\left(\int_{\mathbb{R}}\left(\bar{F}_{1, t}(R-x)-\bar{F}_{1, t}(-R-x)\right) \mathrm{d} \bar{F}_{2, t}(x)\right) \\
& \leq \overline{\mathrm{E}}_{t}\left(I_{1}+I_{2}+I_{3}\right)+\overline{\mathrm{P}}_{t}\left(C_{\eta}^{c}\right),
\end{aligned}
$$

where

$$
\begin{gathered}
C_{\eta}:=\left\{\left|b\left(\tilde{s}_{t}\right)^{2}-m_{0}(L)\right| \leq \eta\right\}, \quad \eta \in\left(0, m_{0}(L)\right), \\
I_{1}:=\mathbb{I}_{C_{\eta}} \int_{\mathbb{R}}\left|F_{1, t}^{*}\left((R-x)^{*}\right)-\Phi\left((R-x)^{*}\right)\right| \mathrm{d} F_{2, t}(x), \\
I_{2}:=\mathbb{I}_{C_{\eta}} \int_{\mathbb{R}}\left|F_{1, t}^{*}\left((-R-x)^{*}\right)-\Phi\left((-R-x)^{*}\right)\right| \mathrm{d} F_{2, t}(x), \\
I_{3}:=\mathbb{I}_{C_{\eta}} \int_{\mathbb{R}}\left(\Phi\left((R-x)^{*}\right)-\Phi\left((-R-x)^{*}\right)\right) \mathrm{d} F_{2, t}(x),
\end{gathered}
$$

where $\Phi$ is the standard Gaussian distribution function.

Now, from the Berry-Esseen inequality (see Theorem 3 with $\delta=1$ in Section 9.1 of Chow and Teich (1997)) we obtain

$$
\begin{aligned}
\overline{\mathrm{E}}_{t}\left(I_{1}+I_{2}\right) & \leq 2 c_{1} \overline{\mathrm{E}}_{t}\left(\frac{\sum_{l=1}^{\tilde{v}_{t}}\left|\pi_{l}\right|^{3} \tilde{\alpha}_{l}^{3} M_{3}(L)}{M_{2}(L)^{3 / 2} b\left(\tilde{s}_{t}\right)^{3}} \mathbb{I}_{C_{\eta}}\right) \\
& \leq 2 c_{1} \frac{M_{3}(L) m_{0}(L)}{M_{2}(L)^{3 / 2}\left(m_{0}(L)-\eta\right)^{3 / 2}} \mathrm{E}_{t}\left(\sum_{l=1}^{\tilde{v}_{t}}\left|\pi_{l}\right|^{3}\right) \\
& =2 c_{1} \frac{M_{3}(L) m_{0}(L)}{M_{2}(L)^{3 / 2}\left(m_{0}(L)-\eta\right)^{3 / 2}} \exp \left(-t\left(1-2 \beta_{3}\right)\right) .
\end{aligned}
$$


The second inequality follows from the fact that, on $C_{\eta}, b\left(\tilde{s}_{t}\right)^{3} \geq\left(m_{0}(L)-\eta\right)^{3 / 2}$, while the equality is derived from (12). Arguing in the same fashion and taking account of the shape of the centered Gaussian density,

$$
\overline{\mathrm{E}}_{t}\left(I_{3}\right) \leq \frac{2 R}{\sqrt{2 \pi}\left(m_{0}(L)-\eta\right)^{1 / 2} M_{2}(L)^{1 / 2}} .
$$

Finally, from the Bienaymé-Chebyshev inequality and (12),

$$
\overline{\mathrm{P}}_{t}\left(C_{\eta}^{c}\right) \leq \frac{1}{\eta^{2}} m_{0}(L)\left(1-m_{0}(L)\right) \mathrm{E}_{t}\left(\sum_{k=1}^{\tilde{v}_{t}} \tilde{\pi}_{k}^{4}\right)=\frac{1}{\eta^{2}} m_{0}(L)\left(1-m_{0}(L)\right) \mathrm{e}^{-t / 4} .
$$

Now, (16)-(18) yield

$$
\begin{aligned}
\overline{\mathrm{P}}_{t}\left(-R \leq \tilde{V}_{t} \leq R\right) \leq & \frac{2 c_{1} L}{M_{2}(L)^{1 / 2}\left(m_{0}(L)-\eta\right)^{3 / 2}} \exp \left(-t\left(1-2 \beta_{3}\right)\right) \\
& +\frac{2 R}{M_{2}(L)^{1 / 2} \sqrt{2 \pi}\left(m_{0}(L)-\eta\right)^{1 / 2}}+\frac{1}{\eta^{2}} m_{0}(L)\left(1-m_{0}(L)\right) \mathrm{e}^{-t / 4},
\end{aligned}
$$

and, in view of the fact that $c_{1}<1$ (see Shiganov (1989)), a new bound can be given for the right-hand side of (19), i.e.

$$
\begin{aligned}
\frac{1}{m_{2}(L)^{1 / 2}}( & \frac{\sqrt{2} R}{\left(m_{0}(L)-\eta\right)^{1 / 2}} \\
& \left.\quad+\frac{2 L}{\left(m_{0}(L)-\eta\right)^{3 / 2}} \exp \left(-t\left(1-2 \beta_{3}\right)\right)+\frac{m_{2}(L)^{1 / 2}\left(1-m_{0}(L)\right)}{\eta^{2}} \mathrm{e}^{-t / 4}\right) .
\end{aligned}
$$

Now, take $L=L_{t}:=\exp \left(t\left(1-2 \beta_{3}\right)\right)$ for any $t$ such that $\left(1-m_{0}\left(L_{t}\right)\right) \leq \eta^{2}$, and choose

$$
R \geq \frac{2}{\left(m_{0}\left(L_{t}\right)-\eta\right)(2-\sqrt{2})}
$$

to obtain the bound

$$
\frac{2 R}{m_{2}(L)^{1 / 2}\left(m_{0}(L)-\eta\right)^{1 / 2}}+\mathrm{e}^{-t / 4} .
$$

Hence, combining the last inequality with (15),

$$
\begin{aligned}
\overline{\mathrm{P}}_{t}\left(\tilde{V}_{t}<-R\right) & =\overline{\mathrm{P}}_{t}\left(\tilde{V}_{t}>R\right) \\
& =\frac{1}{2}\left(1-\overline{\mathrm{P}}_{t}\left(\left|\tilde{V}_{t}\right| \leq R\right)\right) \\
& \geq \frac{1}{2}\left(1-\frac{2 R}{m_{2}\left(L_{t}\right)^{1 / 2}\left(m_{0}\left(L_{t}\right)-\eta\right)}-\mathrm{e}^{-t / 4}\right),
\end{aligned}
$$

which, together with (14), yields

$$
\begin{aligned}
\mathrm{P}_{t}\left(\tilde{V}_{t}<-R\right) \geq & \frac{1}{2}\left(1-\frac{1}{m_{2}(L)^{1 / 2}}\left(\frac{\sqrt{8} R}{m_{0}(L)-\eta}+\mathrm{e}^{-t(8 / 3 \pi-3 / 4)}\right)\right) \\
& +\frac{\mathrm{e}^{-t}}{2}\left(\mu_{0}(-\infty,-R]-\mu_{0}[R,+\infty)\right),
\end{aligned}
$$

and an analogous bound for $\mathrm{P}_{t}\left(\tilde{V}_{t}>R\right)$. This completes the proof of Theorem 1 . 
To prove Corollary 1 it is enough to replace, in the above argument, $\tilde{v}_{t}$ with $n, \pi_{l}$ with $\lambda_{j, n}$, and $L_{t}$ with $L_{n}=\lambda_{(n)}^{-1}$.

The proof of Proposition 1 can be carried out in an analogous way. In addition to the changings mentioned in the proof of the corollary, we have to substitute $y^{*}$ with

$$
\bar{y}^{*}=\frac{y-M_{1}(L) \sum_{j=1}^{n} \lambda_{j, n} \tilde{\alpha}_{j}}{\sqrt{\sum_{j=1}^{n} \lambda_{j, n}^{2} \tilde{\alpha}_{j}^{2} \bar{M}_{2}(L)}}
$$

and $M_{k}(L)$ with

$$
\bar{M}_{k}(L):=\int_{\mathbb{R}}\left|x-M_{1}(L)\right|^{k} \underline{\mu}_{L}(\mathrm{~d} x)
$$

when $k=2,3$. Consequently, we have to make simple adaptations to the inequalities using (13) to obtain $\bar{M}_{2}(L) \geq(1-\eta) m_{2}(L)$, provided that, given $\eta$ in $(0,1), L$ is sufficiently large.

\section{Acknowledgements}

The first author would like to thank University of Pavia for its hospitality at the start of this work. All three authors would like to thank the E. Schroedinger Institute for its hospitality during the completion of this work. Moreover, they are grateful to an anonymous referee for his/her time, comments, and suggestions.

E. Carlen's research was partially supported by US National Science Foundation grant DMS 06-00037. E. Gabetta's research was partially supported by MIUR grant 2006/015821. E. Regazzini's research was partially supported by MIUR grant 2006/134526.

\section{References}

Bobylev, A. V. (1984). Exact solutions of the nonlinear Boltzmann equation and the theory of relaxation of a Maxwellian gas. Teoret. Mat. Fiz. 60, 280-310.

Carlen, E. A., Carvalho, M. C. and Gabetta, E. (2000). Central limit theorem for Maxwellian molecules and truncation of the Wild expansion. Commun. Pure Appl. Math. 53, 370-397.

Carlen, E. A., Carvalho, M. C. and Gabetta, E. (2005). On the relation between rates of relaxation and convergence of Wild sums for solutions of the Kac equation. J. Funct. Anal. 220, 362-387.

Carlen, E. A., Gabetta, E. And Regazzini, E. (2007). On the rate of the explosion of infinite energy solutions of the spatially homogeneous Boltzmann equation. J. Statist. Phys. 129, 699-723.

Chow, Y. S. AND TeICHer, H. (1997). Probability Theory: Independence, Interchangeability, Martingales, 3rd edn. Springer, New York.

CRIMALDI, I. (2002) Convergence results for a normalized triangular array of symmetric random variables. Expo. Math. 20, 375-384.

De FinetTi, B. (1970). Teoria della Probabilità. Einaudi, Torino.

Gabetta, E. ANd RegazzINI, E. (2006a). Some new results for McKean's graphs with applications to Kac's equation. J. Statist. Phys. 125, 947-974.

Gabetta, E. and Regazzini, E. (2006b). Central limit theorem for the solution of the Kac's equation. To appear in Ann. Appl. Prob.

Gnedenko, B. V. And Kolmogorov, A. N. (1954). Limit Distributions for Sums of Independent Random Variables. Addison-Wesley, Cambridge, MA.

KaC, M. (1956). Foundations of kinetic theory. In Proc. 3rd Berkeley Symp. Math. Statist. Prob., 1954-1955, Vol. 3, ed. J. Neyman, University of California Press, pp. 171-197.

KAC, M. (1959). Probability and Related Topics in Physical Sciences. John Wiley, New York.

Khintchine, A. Y. (1935). Sul dominio di attrazione della legge di Gauss. Giorn. Ist. Ital. Attuari 6, 378-393.

McKean, H. P., JR. (1963). Entropy is the only increasing functional of Kac's one-dimensional caricature of a Maxwellian gas. Z. Wahrscheinlichkeitsth. 2, 167-172.

McKean, H. P., JR. (1966). Speed of approach to equilibrium for Kac's caricature of a Maxwellian gas. Arch. Rational Mech. Anal. 21, 343-367. 
McKean, H. P., JR. (1967). An exponential formula for solving Boltzmann's equation for a Maxwellian gas. J. Combinatorial Theory 2, 358-382.

Murata, H. AND TANAKA, H. (1974). An inequality for certain functionals of multidimensional probability distributions. Hiroshima Math. J. 4, 75-81.

Shiganov, I. S. (1989). A note on numerical rate of convergence estimates in central limit theorem. J. Soviet Math. 47, 2810-2816.

TANAKA, H. (1973). An inequality for a functional of probability distributions and its applications to Kac's onedimensional model of a Maxwellian gas. Z. Wahrscheinlichkeitsth. 27, 47-52.

TanaKa, H. (1978). Probabilistic treatment of the Boltzmann equations of Maxwellian molecules. Z. Wahrscheinlichkeitsth. 46, 67-105.

WILD, E. (1951). On Boltzmann's equation in the kinetic theory of gases. Proc. Camb. Philos. Soc. 47, 602-609. 